\title{
A panoramic view of the Milky Way HI gas
}

\author{
Peter M.W. Kalberla*, ${ }^{a}$ Naomi M. McClure-Griffiths ${ }^{b} \dagger$ Jürgen Kerp ${ }^{a} \ddagger$ \\ ${ }^{a}$ Argelander-Institut für Astronomie, Auf dem Hügel 71, D-53121 Bonn, Germany \\ ${ }^{b}$ Australia Telescope National Facility, CSIRO, Marsfield NSW 2122, Australia \\ E-mail: pkalberla@astro.uni-bonn.de, \\ Naomi.Mcclure-Griffiths@csiro.au, jkerpeastro.uni-bonn.de
}

Neutral atomic hydrogen (H I ) traces the interstellar medium (ISM) over a broad range of physical conditions. Its $21-\mathrm{cm}$ emission line is a key probe of the structure and dynamics of galaxies. This line comprises very different temperature and density regimes on all scales, from tens of astronomical units to kiloparsecs. H I is the key element to study the evolution of the ISM in detail. To understand the physics of the ISM and to analyze the interplay between different phases it is mandatory to cover observationally a broad range of scales. But large scale imaging of galaxies, resolving at the same time all these scales is difficult; spatial resolution, as well as sensitivity and the field of view are currently rather limited.

The observational situation is much more favorable if we consider our own galaxy. Two major all sky 21-cm line surveys of the Milky Way will become available soon. The Galactic All Sky Survey (GASS) obtained with the Parkes $64-\mathrm{m}$ telescope $^{\S}$ for the southern hemisphere with a resolution of 16 arcmin is close to completion. The northern extension, the Effelsberg Bonn HI Survey (EBHIS) ${ }^{\mathbb{T}}$ with 9 arcmin resolution, will be available in 2010/2011; we refer to the talks by Kerp and Winkel. Here we discuss briefly the GASS and demonstrate the unprecedented quality of this survey.

The Galactic single dish 21-cm line surveys prepare the ground for future high resolution imaging of the Galactic H I distribution. Using the available short spacing informaton, the Australian Square Kilometre Array Pathfinder (ASKAP) will be capable to generate a truly panoramic view of the Milky Way HI gas distribution with arcsecond resolution for all declinations $<30^{\circ}$. Data from the Widefield ASKAP L-band Legacy All-Sky Blind Survey (WALLABY, see talk by Staveley-Smith) can be used to generate high resolution all sky maps. In comparison to the currently available interferometric International Galactic Plane Surveys (IGPS) the sensitivity will improve by a factor of 10 . Most important is the all sky coverage which will overcome the rather limited spatial coverage of a few degrees around the Galactic plane for the IGPS.

Panoramic Radio Astronomy: Wide-field 1-2 GHz research on galaxy evolution

June 2-5 2009

Groningen, the Netherlands

\footnotetext{
* Speaker.

${ }^{\dagger}$ For the GASS consortium.

${ }^{\ddagger}$ For the EBHIS consortium.

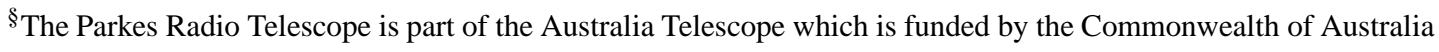
for operation as a National Facility managed by CSIRO

IIBased on observations with the 100-m telescope of the MPIfR (Max-Planck-Institut für Radioastronomie) at Ef-
} felsberg 


\section{The Milky Way galaxy, an outstanding source for ISM research}

Several major Galactic 21-cm line surveys have been released during the past decade [1]. Based on this our knowledge about details in the $\mathrm{H}$ I distribution in our Galaxy is far better than that of other galaxies. To demonstrate observational limitations in galactic research we use M31 as an example. Observing this galaxy with a 1 arcmin beam, we can resolve a linear dimension of $200 \mathrm{pc}$. The situation for more distant galaxies is even worse. Such scales are barely of interest for the physics of the interstellar medium [1].

For the Milky Way the situation is much more favorable. For a 1 arcmin beam the resolution in comparison to M31 is better by at least a factor of 20 for even the most distant regions of the Milky Way. The spatial resolution obtained with large single dish telescopes is comparable to interferometric observations of M31 but in this case the single dish sensitivity is better by a factor of 20. Large scale surveys reach easily a sensitivity of $50 \mathrm{mK}$ which is quite a challenge for synthesis telescopes.

This simplistic comparison demonstrates that the Milky Way galaxy, from observations, is an outstanding source for ISM research. In the following we give first results for the GASS and argue for supplementing single dish observations with high resolution ASKAP observations.

\section{GASS: a high resolution, sensitive, and accurate $21-\mathrm{cm}$ line survey}

The Galactic All Sky Survey was observed with the Parkes 64-m telescope and covers declinations $\delta<1^{\circ}$. The GASS is the most sensitive, highest angular resolution survey of Galactic $\mathrm{H}$ I in the Southern sky [2].

Single dish observations of the Galactic HI line emission suffer from stray radiation originating at the rim of the primary mirror (spill-over) and also from reflections at the feed support legs (straycones). It is therefore mandatory to calculate and eliminate this contribution from the observations. Such corrected GASS data will be available soon, here we demonstrate the superior quality of the forthcoming second GASS data release.

Fig. 1 shows the emission at $v=-30 \mathrm{~km} \mathrm{~s}^{-1}$. The map in the center results after correcting the observations for stray radiation and other instrumental effects. The contribution from the beam pattern which was calculated by convolving the antenna sidelobes with the $\mathrm{HI}$ emission on the sky and which was subtracted from the observations is shown on top of Fig. 1. The obvious patchy structure results from sidelobe contributions that vary with time and season, the patches correspond to individual observing sessions. The line emission after cleaning (center panel) shows no correlations with the observing patches and discloses a rich wealth of faint filaments which were previously hidden. The bottom panel displays data from the Leiden/Arentine/Bonn (LAB) survey [3]. These data have also been corrected for instrumental biases. Previously the LAB was considered to be the most accurate $21-\mathrm{cm}$ line survey but apparently not all of the stray radiation could be eliminated; boxy artifacts correlate also in case of the LAB with observing sessions.

We conclude that the quality of the GASS with a sensitivity of $40 \mathrm{mK}$ is much superior to all other currently available Galactic $21-\mathrm{cm}$ line surveys of the Southern sky. 

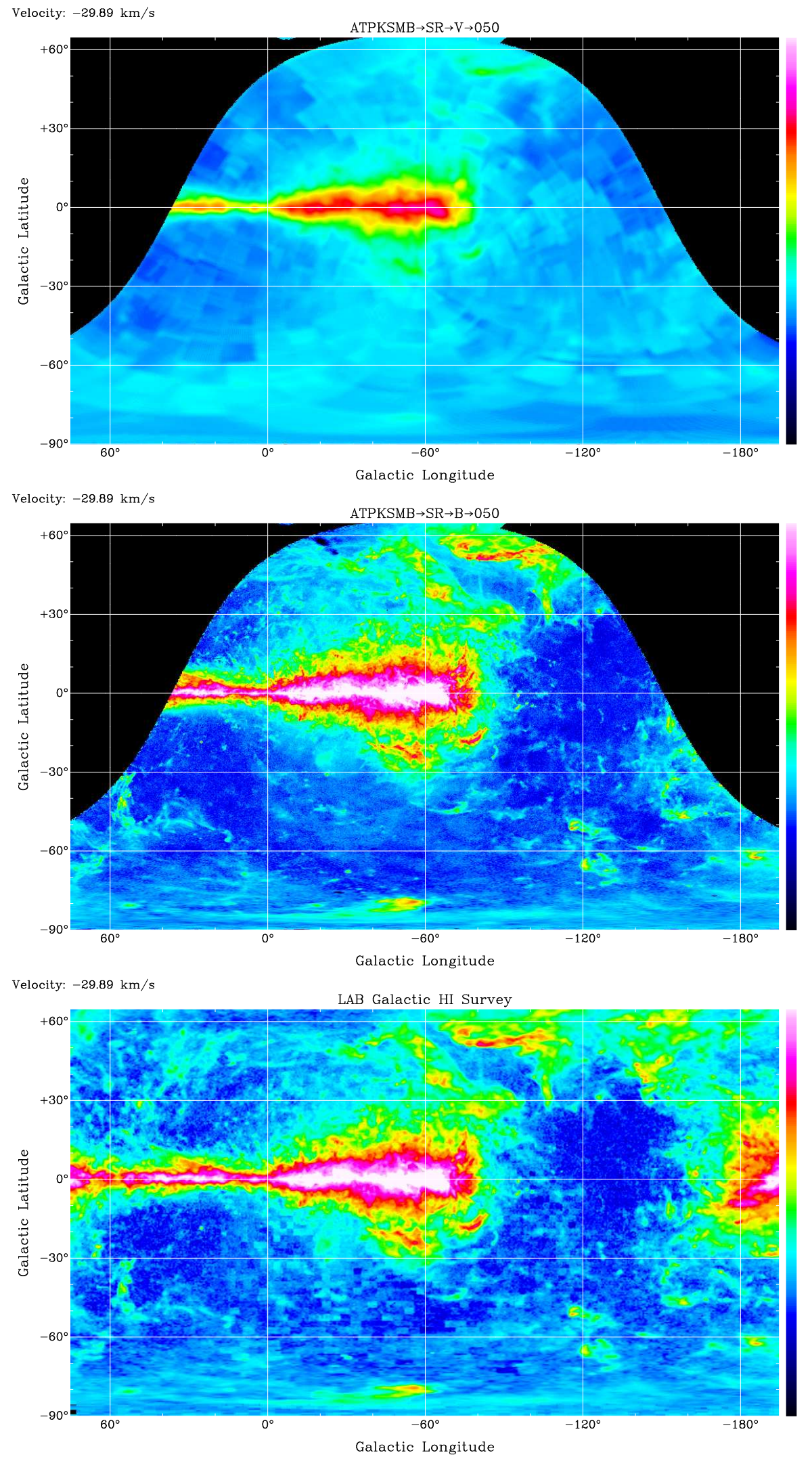

Figure 1: Three channel-maps at $v=-30 \mathrm{~km} \mathrm{~s}^{-1}$. The GASS map is in the middle, stray radiation which was eliminated is on the top. At the bottom the LAB map is shown for comparison. A logarithmic transfer function was chosen to emphasize low brightness temperatures. Black areas indicate positive declinations. 


\section{ASKAP: aiming for panoramic images of the Galactic $\mathrm{H}$ I gas}

Galactic surveys are essential for ISM research and sensitive high resolution single dish surveys like GASS and EBHIS are a major step forward. Still, there is a need for supplementing high resolution data. The $\mathrm{H}$ I line comprises very different temperature and density regimes on all scales, from tens of astronomical units to kiloparsecs. In particular, the analysis of the cold and clumpy component of the multi phase medium on scales of parsecs and below requires interferometer observations. This concerns pressure balance between the different phases and transitions between them. Tiny scale atomic structures do exist with low column densities and linear dimensions of a few hundred or thousand astronomical units. Also little is known about the disk-halo interface and the regulation of the gas flow. If we want to understand galaxy formation and evolution we need first to understand the processes on scales where critical transitions happen.

The new H I surveys will open a new window to the early universe. Future X-ray observatories will have most of their detection power in the soft X-ray energy range below $1 \mathrm{keV}$. This is caused by the large photoelectric absorption cross section of the ISM. The softer the X-ray radiation of interest, the larger the attenuation of the X-rays by the Galactic interstellar medium. The emission of active galactic nuclei at high redshifts $(z \sim 10)$ or the faint emission of clusters of galaxies at moderate redshifts $(3<z<5)$ is shifted to this soft X-ray band. It is not possible to analyze the X-ray data quantitatively without knowing in detail the distribution of the Galactic interstellar medium. High resolution data on scales of arcminutes and below are needed. Our current view through "windows" like the Lockman hole and the Chandra deep-field south may result in a tunnel vision of the early universe, strongly biasing our knowledge towards specific large scale structures.

ASKAP offers a unique chance to supplement the GASS and EBHIS surveys with high resolution interferometric data. We propose to use data from the Widefield ASKAP L-band Legacy All-sky Blind surveY (WALLABY, presented by Staveley-Smith) to obtain a complete unbiased high quality database at arcsecond resolution. For a synthesized beam of 60 arcseconds a sensitivity of $300 \mathrm{mK}$ is feasible, a factor of 10 improvement with respect to the currently available IGPS data. Such a Galactic ASKAP survey would cover the southern sky up to declinations $\delta<30^{\circ}$. At arcsecond resolution the ASKAP images would provide a truly panoramic view of the Galactic H I gas over a broad spectral window of $-400<v<400 \mathrm{~km} \mathrm{~s}^{-1}$ with a velocity resolution of 4 $\mathrm{km} \mathrm{s}^{-1}$.

ACKNOWLEDGEMENTS: PK acknowledges support from Deutsche Forschungsgemeinschaft, grant KA1265/5, JK from grant KE757/7.

\section{References}

[1] Kalberla, P. M. W., Kerp, J. The H I Distribution of the Milky Way, 2009. Annu. Rev. Astron. Astrophys. 47, 27

[2] McClure-Griffiths, N. M., et al. The Parkes Galactic All-Sky Survey. I. Survey Description, Goals, and Initial Data Release, 2009, APJS 181, 398 [arXiv : 0901.1159 ]

[3] Kalberla, P. M. W. et al., The Leiden/Argentine/Bonn (LAB) Survey of Galactic HI. Final data release of the combined LDS and IAR surveys with improved stray-radiation corrections, 2005, A\&A 440, 775 [arXiv:astro-ph/0504140] 